\title{
Application of power matching deviation coefficient in drying stage production control of Dadu River cascade hydropower stations
}

\author{
Fuzhi Wang ${ }^{1, a}$, Zujian Zou ${ }^{1}$ and Qingxiang Zhong ${ }^{1}$ \\ ${ }^{1}$ Dadu River Hydropower Development Co., Ltd., Chengdu 610041, China
}

\begin{abstract}
The economic indicators of cascade hydropower stations are the main technical means in the current economic operation management, but there are management loopholes in various economic indicators. Aiming at the particularity of the operation of cascade hydropower stations in dry season and the new form of grid reform, combined with the characteristics of various economic indicators, the power matching deviation coefficient is proposed to hedge the loophole of comprehensive water consumption rate, and the price difference is used to balance the spread between cascade hydropower stations. This indicator can correctly guide production and operation personnel to tap potential and increase efficiency and ensure healthy competition while ensuring the overall economic operation. The evaluation system has a good effect in the actual use of the Dadu River cascade hydropower stations, and has obvious promotion effect on the production control of the cascade hydropower stations.
\end{abstract}

\section{Introduction}

In the dry season, when the amount of water in the cascade reservoir is certain and the natural incoming water is in a stable base flow, there is basically no abandonment of water. The main task of the hydropower station production operation is to reduce the comprehensive water consumption rate of the cascade hydropower stations as much as possible, and to maximize the quantification. The water can be converted into electrical energy to maximize the benefits of power generation. When the cascaded power stations maintain the theoretical optimal operating water level and maintain the power generation output corresponding to the incoming water, the ideal operating state can be achieved. At present, economic indicators of cascade hydropower stations are widely used for optimizing calculation or evaluation of production operation process , especially the comprehensive water consumption rate index during dry season, which can reflect the whole cascade generation operation as a whole, and can also evaluate the power generation capacity of cascade hydropower stations through scientific calculation. Through the analysis of the water consumption rate of each power station inside the cascade, the efficiency of the cascade power generation can be optimized.

However, due to the variability and complexity of reservoir operation, power market demand and power grid transmission, hydropower stations often have to adjust the upstream flow of water to meet changes in grid power flow. In addition, the actual scheduling production process is an uninterrupted process, which needs to be completed by the rotation between several scheduling teams. The hydraulic and power balance of the upstream and downstream power stations needs to be adjusted by the dispatching personnel according to the actual situation. Even if an advanced economic operation model of cascade hydropower stations is established, the actual operation is affected by the game between supply and demand of the cascade hydropower stations and the behavior and preferences of the dispatchers ${ }^{[1]}$, and it is difficult to achieve the optimization objectives. In particular, the short-term scheduling objectives and intermittent statistical evaluation indicators of scheduling teams are different from the overall objectives of long-term optimization. It is difficult to form cooperative behaviors without economic constraints with additional constraints ${ }^{[2]}$. Internal bad competition is likely to cause deviations from scheduling objectives.

The comprehensive index can not reflect the specific situation of each link between the cascade hydropower stations. Starting from the characteristics of the cascade hydropower stations and electric power production, a scientific and reasonable management index is designed to feedback the connection status between the cascade hydropower stations, and the bad conduct of the dispatching operators are constrained, Behavior to ensure the overall efficiency of cascade hydropower stations have great significance to promote the economic operation management.

\footnotetext{
${ }^{a}$ Corresponding author: shigau@163.com
} 


\section{Power production characteristics and management objectives of cascade hydropower stations}

The electric power production process of cascade hydropower stations has the following characteristics:

Real-time: Due to the non-storage of electrical energy, the production and consumption of electricity occurs almost simultaneously. In the production process, it is necessary to take the initiative to carry out the inter-plant and in-plant economic operation according to the load demand, the water condition of the power station and the condition of the equipment.

Continuity: The power production process is uninterrupted, and the flow of natural rivers is also continuous. Hydropower stations without regulation performance need to convert water energy into electricity for the purpose of timely.

Rotation: Human biological structure and labor law determine that hydropower production needs to shift between several teams, and dispatchers' working hours are intermittent. The daily production process generally requires three scheduling teams to be rotated, five teams-three shifts mode is popular in most of the cascade control center. While encouraging cooperation, it is necessary to stimulate work enthusiasm through competition.

Seasonality: All provinces have their own measures of electricity prices in flood and dry season, and employ time-of-use electricity prices. At present, Sichuan Province implements different electricity prices for flood seasons and dry periods, electricity prices are relatively high during dry season because only quantitative water resources. It is necessary to maximize the efficiency of converting water into electricity, and to avoid water plants from abandoning water or operating at low efficiency.

Relevance: There are close hydraulic and power links between cascade hydropower stations, and the demand for water resources and the competition in the electricity market exist simultaneously. It is necessary to coordinate the production of upstream and downstream power stations scientifically and rationally to improve the overall power generation performance while reducing operational risks.

The above five characteristics determine that the power production process of hydropower stations want to improve the economic operation level, it should establish a dynamic balance at the competition level.

\section{Advantages and disadvantages of cascade water consumption rate}

After canceling the peak-to-valley electricity price, the factors affecting the electricity price of the electricity generation in the power generation value are excluded, and the power generation income depends mainly on the amount of power generation, therefore, the primary goal of power generation operation is to minimize the overall water consumption rate of the cascade hydropower stations. Usually the comprehensive water consumption rate is used for the evaluation of the operation of the entire cascade, which is effective for macro management objectives. In the management of the specific operation process, the evaluation of the comprehensive water consumption rate during the operation team's work time has pros and cons.

This economic indicator refers to the comprehensive water consumption rate of a cascade hydropower stations during the work of a team, and the calculation method is as follows:

$$
\mu_{i}=\frac{W_{i}}{E_{i}}
$$

Where: $\mu$ is the cascade comprehensive water consumption rate, the unit is $m^{3} / \mathrm{kWh}$; $W$ is the total water consumption for power generation of the cascade hydropower stations, the unit is ten thousand $\mathrm{m}^{3} ; E$ is the total power generation of the cascade hydropower stations, the unit is ten thousand $k W h ; i$ is team $i, i=1,2$, $3 \ldots$

Advantages: It can reflect the economic operation status of the entire cascade power station. This parameter summarizes the results of the production operation and points out the direction of the production operation.

Disadvantages: The comprehensive water consumption rate is a macro indicator of the entire power generation process. The production time between the operating teams is intermittent and interconnected, the water consumption rate and the water flow arrival time is different between the power stations in the cascade. When the power of a power station with a small water consumption rate is increased by depressing the power generation of a large water consumption rate during a duty period, the comprehensive water consumption rate of the team can be greatly reduced statistically. At this time, the next team of the succession is often faced with the small water consumption rate power plants have little water consumption, and the power plants with large water consumption rate have a high risk of water abandonment, and have to ask for more power generation. In the end, all teams only pursue the statistically comprehensive water consumption rate of the team and leave the bad situation formed to the next team, which make a vicious circle. Managers will face huge production risks and obtain poor macro indicators at any time.

\section{Power matching deviation coefficient of cascade power stations}

According to the advantages and disadvantages of the comprehensive water consumption rate index and its important significance in the comprehensive benefits of cascade hydropower stations, it is necessary to exert its advantages to improve the overall economic operation level, and to establish a defect constraint mechanism from the details to supervise the behavior of dispatchers and jointly improve the whole In the comprehensive economic operation level of the cascade. This paper proposes the Power Matching Deviation Coefficient (PMDC) to balance the defects of the comprehensive 
water consumption rate, and to Protect the joint operation of cascade hydropower stations.

From the actual operating parameters of the cascade hydropower stations and the analysis of hydrological and hydraulic characteristics of the river basin, the proportion of power generation in the relatively stable operation between the cascade hydropower stations can be calculated, and the maximum water energy utilization or maximum power generation income can be obtained by the relevant optimization model function. The power ratio parameter is the theoretical optimal or planned optimal of the decision target, and the actual operation cannot be achieved. There must be a certain positive and negative deviation from the value. This difference can reflect the distance between the actual operation of the cascade power station and the management target. At the same time, it can be analyzed whether the power generation strategy used in actual operation is biased or deviating, thus reflecting whether the power generation strategy adopted by the teams is reasonable.

\subsection{Proportion of electricity in upstream and downstream power station}

When the power stations are in good operating condition, the power generation output of the cascade power stations is after the hydraulic factors are fully considered. The ratio of the theoretical equilibrium output of the upper and lower power stations is the inverse ratio of the water consumption rate.. The calculation method of the ratio is:

$$
\partial_{n}=\frac{\exists \mu_{n+1}}{\exists \mu_{n}}
$$

Where: $\partial_{n}$ is the power ratio coefficient of the $n$th power station; $\exists \mu_{n}$ is the water consumption rate of the nth power station, the unit is $m^{3} / k W h ; \exists \mu_{n+1}$ is the water consumption rate of power station $n+1$.

From the perspective of scheduling strategy, the water consumption rate pursued by management can be calculated by the historical operation data statistics optimal value, or calculated according to the hydropower station operation chart ${ }^{[3]}$, or the theoretical optimal water consumption rate can be derived from the formula: $N=g \eta Q H$.

$$
\exists \mu=\frac{3600}{g \eta H}
$$

where, $\exists \mu$ is the water consumption rate; $g$ is the gravity acceleration, generally $9.81 ; \eta$ is the comprehensive conversion efficiency of the turbine generator, which can be obtained by using the generator set factory parameters, statistical data or measuring device ${ }^{[4,5]} ; H$ is the generating water head, the unit is $m$, for the convenience of calculation can also be replaced by the actual running average head.

When the market conditions are more stringent, the total power generation of the cascade is controlled. If the enterprise pursues profit maximization, it needs to consider the factors of electricity price, and appropriately sacrifice the water consumption rate and power generation of the power station with low electricity price. At this time, the power ratio coefficient is:

$$
\partial_{n}=\frac{\beta_{n} \exists \mu_{n+1}}{\beta_{n+1} \exists \mu_{n}}
$$

where, $\beta$ is the on-grid price of the power station.

\subsection{Power Matching Deviation Coefficient (PMDC)}

In the cascade hydropower stations, large-scale control hydropower stations are often used as the regulating center of cascade hydropower stations. The regulation performance of the power plants above the seasonal regulation performance is large, the operating parameters are excellent, and the short-term impact by the upstream power stations is small. This power station does not have the meaning of synchronous operation. The power station with daily adjustment performance generally operates in the manner of maintaining the highest head to maximize the economic operation level. It is better to use a step-by-step ratio to maintain the head of the cascade power stations. The runoff power station should also use the synchronous ratio of the upstream power station to stabilize the operation of the power station and prevent the water from being abandoned. For example, if the power station $m$ of the $N$ power stations from the upstream to the downstream is a control power station, the matching of the power stations $m-1$ and $m$ is not calculated, and only the ratio between the power station $m$ and the lower power station is calculated.

The difference between the proportion of electricity generated by the actual production operation of each power station and the ratio can reflect the degree of cooperation between the upper and lower power stations. When the actual operating parameter minus the theoretical value is a positive number, it indicates that the power station in the upper level has super-power generation or the under-power generation of the next-level power station, it will increase the risk of water abandonment. Negative deviations are just the opposite. The downstream power stations are forced to lower the water head and the water consumption rate of power generation increases.

A team during duty, the calculation method of the difference between the n-stage and the next-stage power station power matching coefficient is:

$$
\Delta_{i, n}=\frac{E_{i, n}}{E_{i, n+1}}-\partial_{n}
$$

Where: $\Delta_{\mathrm{i}, \mathrm{n}}$ is the matching deviation between the $\mathrm{n}$ and the next stage power station, and $\Delta_{\mathrm{i}, \mathrm{n}}$ may be positive or negative; $E_{i, n}$ is The actual power generation of the n-stage power station during the work of team $i ; E_{i, n+1}$ is the actual power generation of the $n+1$-stage power station during the work of the team $i$.

Since the flow of water has a confluence time between the power stations, when calculating the proportion of actual power generation, the statistical time 
difference should be considered as appropriate.

\subsection{Comprehensive PMDC of cascade power stations}

According to the characteristics of the upstream and downstream power stations, the positive and negative deviation values are scaled by different frequencies, selectively induced to guide the working direction of the operating personnel, and the unit of the deviation value is converted into a positive number for comparative analysis of the uniform deviation.

Considering the actual production requirements, the calculated values of the matching coefficients are weighted, scaled, and unit converted to obtain a comprehensive power matching deviation coefficient of the dispatch team.

The calculation method of the comprehensive power matching deviation coefficient of a team is:

$$
\delta_{i}= \begin{cases}\sum_{n=1}^{N}\left(K_{1}, n \Delta_{n}\right), \Delta_{n}<0 \\ \sum_{n=1}^{N}\left(K_{2, n} \Delta_{n}\right), \Delta_{n} \geq 0\end{cases}
$$

Where: $i$ is the team $i ; N$ is the number of step hydropower stations; $n$ is the $n$-stage power station, $n=1$, $2,3 \ldots N ; K_{1, n}$ is the scaling rate indicating the negative deviation, It is a negative number; $K_{2, n}$ is a scaling rate indicating a positive deviation and is a positive number.

\section{Rating method}

The performance of the score should be based on the base plus variable. Therefore, the score difference of each economic indicator should be limited to a certain range of values, so that the overall difference is within a reasonable range, so as to serve as a reference for rewards and assessment.

\subsection{Reduced index}

For indicators such as comprehensive water consumption rate, rotating standby time, and head loss, the smaller the control target is, the better the economic indicator is. Those economic indicators that aim to reduce their value use the following scoring methods:

$$
F \emptyset_{i}=F \varnothing-\left(1-\frac{\emptyset_{\max }-\emptyset_{i}}{\emptyset_{\max }-\emptyset_{\min }}\right) \times f \varnothing
$$

Where: $F \emptyset_{i}$ is the score of the indicator of the scheduling team $i ; F \emptyset$ is the full score of this index; $\emptyset_{i}$ is the index of the scheduling team $i ; \emptyset_{\max }, \emptyset_{\min }$ are the maximum and minimum values of the teams on the $\varnothing$ indicator; $f \varnothing$ is the maximum allowable difference between the optimal team and the worst team.

The integrated PMDC is calculated by the positive and negative conversion statistics of the formula (6). The closer the value is to 0 , the better. Therefore, the smaller the integrated PMDC value, the better, and it is also a reduction index.

\subsection{Increased index}

For indicators such as power generation income, the higher the control target is, the better, the score is as follows:

$$
F S_{i}=F S-\left(1-\frac{S_{i}-S_{\min }}{S_{\max }-S_{\min }}\right) \times f S
$$

Where: $F_{S}$ is the index score of the team i; FS is the full score of the power generation income index; $S_{i}$ is the statistical index of the team i; $S_{\max }$ and $S_{\min }$ are the maximum and minimum values of the statistical indicators of the teams; fS is The maximum difference allowed between the optimal team and the worst team.

Due to the large number of conventional economic indicators of cascade hydropower stations, the indicators can be statistically scored according to the needs, and the scores of each item are added together to become a comprehensive score of the operational team for reward or assessment. It is recommended to increase the evaluation of other economic indicators according to the needs of production management based on the selection of the key economic indicators listed in this paper.

\subsection{Score allocation and reward assessment mechanism}

According to the control significance of each indicator, different scores are assigned to each index, and the primary and secondary points are specified for the production and operation personnel. The secondary economic indicators are optimized on the basis of strengthening the production focus. For example, the main indicator of economic dispatch during the dry season is the comprehensive water consumption rate, and its score accounts for the vast majority of the total score; The auxiliary indicators such as rotating standby time and unit efficiency have little influence on economic dispatch. Due to the rigidity of the power grid system, the subjective effect of dispatcher control is small, mainly to urge the dispatchers to reduce unnecessary losses, its score is generally not large, but it has a certain effect on the accumulation of total scores. Figure 1 shows the approximate distribution of a score for reference only.

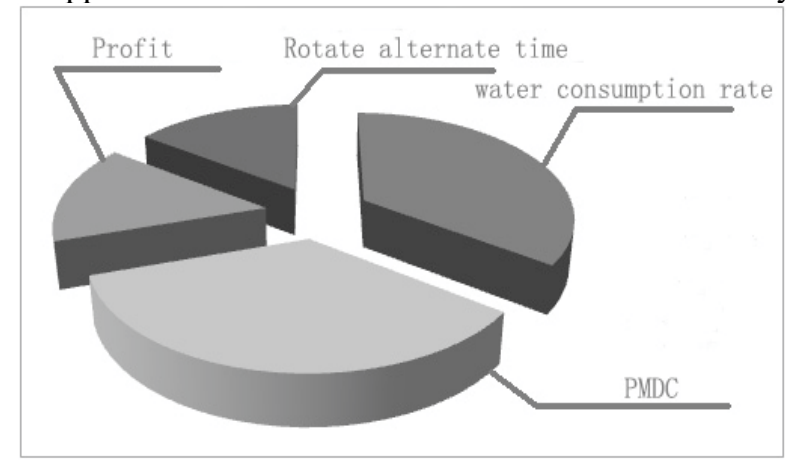

Figure 1. Fractional weight reference map.

The assessment mechanism can be based on the score to issue performance pay. According to the completion of the comprehensive indicators, the difference rewards and punishments is able to evaluate according to the team score. 


\section{Implementation and application}

\subsection{Cascade power stations overview}

The Dadu River Central Control Center is the economic operation center of the Dadu River cascade hydropower stations. At present, it has completed the remote centralized control and unified dispatch of the main stream power plant, including Dagangshan, Pubugou, Shenxigou, Zhentouba, Gongzui and Tongjiezi. There are 6 hydropower stations, 29 remote control units with an installed capacity of 9.05 million kilowatts. The basic adjustment performance parameters of each power station are shown in Table 1 below.

Table 1. Basic parameters of cascade hydropower stations

\begin{tabular}{ccccc}
\hline $\begin{array}{c}\text { Step } \\
\text { number }\end{array}$ & Station name & Adjustment capability & $\begin{array}{c}\text { Installed capacity } \\
(\mathrm{MW})\end{array}$ & $\begin{array}{c}\text { Adjustable reservoir capacity } \\
\left(\text { Million m }^{3}\right)\end{array}$ \\
\hline 1 & Dagagnshan & Weekly & 2600 & 117 \\
2 & Pubugou & Seasonal & 3600 & 3894 \\
3 & Shenxigou & Runoff & 660 & 8 \\
4 & Zhentouba & Runoff & 720 & 12 \\
5 & Gongzui & Daily & 770 & 86 \\
6 & Tongjiezi & Daily & 700 & 48 \\
\hline
\end{tabular}

It can be seen from Table 1 that the Pubugou power station belongs to the mid-stream regulating power station. According to the design content of Section 3.2, the upstream Dagangshan power station does not carry out PMDC evaluation, and it is used as a single power station for conventional economic indicators evaluation. The cascade power station downstream of the Pubugou has little regulation performance, and the comprehensive PMDC evaluation should be carried out to obtain the cascade comprehensive score for the evaluation between the teams.

\subsection{Indicator and statistical cycle}

The centralized control center of the cascade hydropower stations in the Dadu River Basin is implemented with five teams and three shifts. The daily production process requires three scheduling teams to be rotated, and the other two scheduling teams are take turns to rest. Sichuan Province implemented the same electricity prices in dry season and cancelled time-of-use electricity prices.

The centralized control center of the cascade hydropower stations in the Dadu River Basin divides the economic indicators of the dry season into three categories and five indicators. The first category is the key indicators, including the comprehensive water consumption rate and the power matching deviation coefficient; the second category is the important indicators, including the rotating standby time and power generation efficiency; the third category is the auxiliary index. Specific indicator weights can be set according to actual needs.

In order to enhance the comparability of the evaluation indicators between the various scheduling teams, the number of working days of each scheduling team is consistent during the statistical period. For example, in the shift mode of five-team three-shift, the first shift is 15 days, and the working days for each team within 15 days are 3 white shifts, 3 medium shifts, and 3 night shifts. Therefore, the statistical period is an integer multiple of 15 days.

\subsection{Implementation Effect}

The centralized control center of the cascade hydropower stations in the Dadu River Basin will release the results of the competition every month, and will be included in the monthly performance appraisal and monthly special awards to make the monthly assessment. The employees pay attention to the indicators every day. The department often organizes and exchanges the experience of optimizing power generation in combination with the index competition, forming a good atmosphere of "comparing, learning, catching up, helping and super".

Table 2 below is a statistical table of some economic indicators for February of a certain year:

Table 2. February scheduling indicator statistics

\begin{tabular}{ccccccccccc}
\hline & $\begin{array}{c}\text { Comprehensive } \\
\text { water } \\
\text { Team }\end{array}$ & $\begin{array}{c}\text { consumption } \\
\text { rate } \\
\left(\mathrm{m}^{3} / \mathrm{kWh}\right)\end{array}$ & Score & PMDC & Score & $\begin{array}{c}\text { Unit } \\
\text { efficiency } \\
(\%)\end{array}$ & Score & $\begin{array}{c}\text { Rotate } \\
\text { alternate } \\
\text { time } \\
(\mathrm{h})\end{array}$ & $\begin{array}{c}\text { Score } \\
\text { Ra }\end{array}$ & $\begin{array}{c}\text { Total } \\
\text { score } \\
\text { nk } \\
\text { in } \\
\mathrm{g}\end{array}$ \\
\hline 1 & 5.987 & 39.32 & 0.32 & 37.88 & 87.64 & 9.36 & 33.1 & 9 & 95.55 & 2 \\
2 & 6.114 & 36.81 & 0.31 & 38.19 & 87.79 & 9.59 & 13.4 & 9.92 & 94.51 & 4 \\
3 & 5.953 & 40 & 0.40 & 35.4 & 87.99 & 9.93 & 11.7 & 10 & 95.34 & 3 \\
4 & 6.018 & 38.72 & 0.11 & 39.35 & 88.03 & 10 & 19.2 & 9.65 & 97.72 & 1 \\
5 & 6.155 & 36 & 0.20 & 39.64 & 87.44 & 9 & 21.8 & 9.53 & 94.17 & 5 \\
Cascade & 6.043 & $/$ & 0.27 & $/$ & 87.75 & $/$ & 99.2 & $/$ & $/$ & $/$ \\
\hline
\end{tabular}

From the data of Table 2, the team three integrated water consumption is the best, but its PMDC is the worst.
There is obvious bias in the dispatching operation process of this team. It is unreasonable to analyze the 
scheduling process by the power distribution data of the cascade power station. Therefore, the PMDC value can reflect whether a scheduling team optimizes the water consumption rate of the hydropower station while taking into account the benefits of the entire cascade, and its scoring method can effectively balance this hidden scheduling problem.

\section{Conclusion and suggestion}

The use of PMDC indicators of cascade power stations can hedge the shortcomings of economic indicators such as comprehensive water consumption rate, comprehensively affect the impact of power station price differences on income, and play a good role in promoting the economic operation and benign competition control of the cascade hydropower stations in dry seasons. The guiding role is good in the actual production operation of the Dadu River cascade power station. The main role is as follows:

(1) The PMDC index of the cascade power station comprehensively balances the impact of the water consumption rate and electricity price difference between the cascade hydropower stations on the income, and has a good guiding effect on the production operation.

(2) The relevant achievements can be updated in real time, and the production strategy of each dispatch team can be analyzed at any time to facilitate control. The statistical method is fair and equitable, which guarantees healthy competition and facilitates the economic operation of cascade hydropower stations.

(3) The evaluation system produced by this method is in line with the characteristics of the region, and has a comprehensive control over the production process, which can actively promote the enthusiasm of employees.

At the same time, there are some problems and points that need to be paid attention to during the implementation process:

(1) The deviation value needs to be unitized, frequency scaled, and weighted to form unified comparison data. The fitting of relevant parameters requires a large amount of data analysis and continuous debugging to achieve the expected results.

(2) The performance and hydraulic connections of cascade hydropower stations in various river basins are complex and diverse. It is necessary to select suitable power stations for power supply ratio. Some runoff power plants with no regulation performance are mainly for safety. If the safety can not be guaranteed, they need to be excluded from the statistical scope, and require professional intervention with comprehensive analysis.

(3) There are inevitable deviations in the theoretical parameters of each power station, and there are systematic errors in the calculation, which have a certain impact on the results.

(4) The more the number of cascade hydropower stations, the more complicated the calculation process, the cascade hydropower stations can simplify the calculation process to meet its own needs as needed.

\section{References:}

1. Arman Ganji,Davar Khalili,Mohammad Karamouz. Development of stochastic dynamic Nash game model for reservoir operation. I. The symmetric stochastic model with perfect information[J]. Advances in Water Resources, 528-542(2006)

2. Arman Ganji,Mohammad Karamouz,Davar Khalili. Development of stochastic dynamic Nash game model for reservoir operation II. The value of players' information availability and cooperative behaviors[J]. Advances in Water Resources, 157-168(2006)

3. Jianxia Chang, Yunyun Li,Meng Yuan,Yimin Wang. Efficiency evaluation of hydropower station operation: A case study of Longyangxia station in the Yellow River, China[J]. Energy, 23-31(2017)

4. H K Zhang,Q J Chen,D M Liu,H Y Wang,Y F Jing,W $Y$ Wang. Energy efficiency test and analysis of large hydro-generators based on economic evaluation[J]. IOP Conference Series: Earth and Environmental Science,2018,163(1).

5. M.M. Cordova,E.C. Finardi,F.A.C. Ribas,V.L. de Matos,M.R. Scuzziato. Performance evaluation and energy production optimization in the real-time operation of hydropower plants $[\mathrm{J}]$. Electric Power Systems Research, 201-207(2014) 